\section{SciElO Saúde Pública: o desempenho dos Cadernos de Saúde Pública e da Revista de Saúde Pública}

\author{
SciELO Public Health: the performance of \\ Cadernos de Saúde Pública and \\ Revista de Saúde Pública
}

\author{
${ }^{1}$ Faculdade de Ciências \\ Médicas da Santa Casa de \\ São Paulo, São Paulo, Brasil. \\ Correspondência \\ R. B. Barata \\ Departamento de Medicina \\ Social, Faculdade de Ciências \\ Médicas da Santa Casa de \\ São Paulo. \\ Rua Dr. Cesário Motta Jr. 61 \\ São Paulo, SP \\ 01221-020, Brasil. \\ rita.barata@fcmscsp.edu.br
}

\section{Abstract}

The aim of this paper was to analyze two Brazilian scientific journals included in the SciELO Library of Public Health, using a group of bibliometric indicators and scrutinizing the articles most viewed. Cadernos de Saúde Pública was accessed 3,743.59 times per month, with an average of 30.31 citations per article. The 50 articles most viewed (6.72 to 524.5 views) were mostly published in Portuguese (92\%). $42 \%$ were theoretical essays, $20 \%$ surveys, and $16 \%$ descriptive studies. $42 \%$ used argumentative techniques, $34 \%$ quantitative techniques, $18 \%$ qualitative techniques, and 6\% mathematical modeling. The most common themes were: health and work (50\%), epidemiology (22\%), and environmental health (8\%). Revista de Saúde Pública was accessed 1,590.97 times per month, with an average of 26.27 citations per article. The 50 articles most viewed (7.33 and 56.50 views) were all published in Portuguese: $46 \%$ were surveys, $14 \%$ databases analysis, and $12 \%$ systematic reviews. Quantitative techniques were adopted in $66 \%$ of such articles, while mathematical modeling was the same as observed in Cadernos de Saúde Pública, as were qualitative techniques. The most common themes were health services organization (22\%), nutrition (22\%), health and work (18\%), epidemiology (12\%), and environmental health (12\%).

Open Access; Periodicals; Virtual Libraries; Indexes
Rita Barradas Barata ${ }^{1}$

\section{Introdução}

A pesquisa científica é uma atividade que deve satisfazer a três características básicas: ser socialmente relevante, ou seja, seus resultados devem encontrar cedo ou tarde aplicação na solução de problemas humanos; ser ética, ou seja, acima dos interesses científicos deve estar a preservação da dignidade humana; e ser pautada no mérito, isto é, ser conduzida com rigor metodológico para que produza conhecimentos verdadeiros 1,2,3

A garantia da relevância social será dada pelo impacto que os conhecimentos produzidos venham a ter na transformação da realidade. A adequação ética será garantida pela submissão ao julgamento feito pela sociedade acerca dos procedimentos dos cientistas. A produção de conhecimentos verdadeiros depende da correta aplicação do método, mas também do julgamento entre os pares, ou seja, a verdade é construída no interior da comunidade da qual o cientista faz parte ${ }^{1}$.

A publicação dos resultados das pesquisas em periódicos científicos busca cumprir essa dupla função: submeter os conhecimentos produzidos ao julgamento dos pares, e criar uma comunidade de interesses em torno de determinado objeto de investigação. Para cumprir esses objetivos os periódicos necessitam contar com um sistema adequado de "peer-review" e possibilitar a divulgação ampla dos resultados, facilitando desta 
forma a apropriação pelos outros membros da comunidade 1 .

Volpato \& Freitas ${ }^{4}$ afirmam que apesar dos encontros e eventos científicos possibilitarem a divulgação rápida de informações científicas, apenas a divulgação formal por meio das publicações científicas fornece o aval e a credibilidade necessários para que a informação passe a ser incorporada.

Gomes \& Santos 5, referindo outros autores, informam que os periódicos científicos possuem três funções principais: o registro público do conhecimento; conferir prestígio e reconhecimento tanto aos autores quanto aos editores, referees e assinantes; e disseminação da informação.

É em relação a essa última função de disseminação da informação que nos propomos a analisar os dois periódicos selecionados.

SciELO Saúde Pública é uma biblioteca eletrônica on-line de revistas científicas em saúde pública publicadas em países ibero-americanos. Até 2003 estavam incluídos sete periódicos: Bulletin of World Health Organization, Revista Panamericana de Salud Pública, Salud Pública de México, Revista Española de Salud Pública, Gaceta Sanitaria, Revista de Saúde Pública e Cadernos de Saúde Pública. As publicações incluídas há mais tempo eram a Revista de Saúde Pública e a Revista Panamericana de Salud Pública, ambas introduzidas em 1998. Em 1999 foram incorporadas a Revista Española de Salud Pública, Salud Pública de México e Cadernos de Saúde Pública. Em 2001, o Bulletin of the World Health Organization passou a fazer parte da biblioteca e em 2002, a Gaceta Sanitaria. Atualmente conta ainda com a Revista de Salud Pública (Colômbia).

Além de facilitar o acesso e ampliar a divulgação de periódicos produzidos por países em desenvolvimento, a biblioteca eletrônica possui um sistema de estatísticas que permite aos editores das revistas científicas e aos estudiosos da ciência analisar diferentes aspectos das publicações.

O objetivo deste artigo é analisar o desempenho dos dois periódicos brasileiros incluídos na biblioteca SciELO Saúde Pública, por meio de um conjunto de indicadores e análise dos artigos mais acessados.

\section{Metodologia}

Os periódicos incluídos nessa biblioteca virtual têm as seguintes características gerais:

Bulletin of the World Health Organization: publicado mensalmente pela Organização Mundial da Saúde, procurando "estimular o debate sobre políticas e práticas de saúde pública baseadas nas melhores evidências disponíveis, ao mesmo tempo em que encoraja elos mais estreitos entre a investigação científica e o desafio de ajudar a população a ter uma vida mais saudável" (http://www.scielosp.org/revistas/bwho/paboutj.htm).

Cadernos de Saúde Pública: publicação bimestral até 2006, quando passa a ser mensal, da Escola Nacional de Saúde Pública Sergio Arouca, da Fundação Oswaldo Cruz, voltada para a publicação de "artigos originais que contribuam para o estudo da saúde pública em geral e disciplinas afins, tais como a epidemiologia, parasitologia, microbiologia, nutrição, saúde ambiental, ciências sociais aplicadas à saúde, dentre outras" (http:// www.scielosp.org/revistas/csp/paboutj.htm).

Gaceta Sanitaria: publicação bimestral da Sociedad Española de Salud Pública y Administración Sanitaria, contendo "artigos originais e outras formas de comunicação referentes a todos os âmbitos da saúde pública e da administração sanitária, incluindo epidemiologia, prevenção e promoção de saúde, gestão e avaliação das políticas e serviços, economia da saúde, saúde ambiental e saúde internacional" (http://db.doyma. es/cgi-bin/wdbcgi.exe/doyma/mrevista).

Revista de Saúde Pública: publicada a cada dois meses pela Faculdade de Saúde Pública da Universidade de São Paulo, contendo resultados de "pesquisas originais, revisões, comentários e notas científicas no campo da saúde pública com ênfase em epidemiologia" (http://www.scielosp. $\mathrm{org} / \mathrm{revistas} / \mathrm{rsp} /$ paboutj.htm).

Revista Española de Salud Pública: publicada a cada dois meses pela Dirección General de Salud Pública del Ministerio de Sanidad y Consumo da Espanha, contendo artigos em espanhol para "divulgar pesquisas originais e estudos técnicos de profissionais de saúde pública do país, assim como trabalhos e documentos estrangeiros de interesse para a saúde pública espanhola" (http:// www.scielosp.org/revistas/resp/paboutj.htm).

Revista Panamericana de Salud Pública: publicação mensal da Organização Panamericana da Saúde, lançada em 1997 em substituição ao Boletín de la Oficina Sanitaria Panamericana, com artigos de "pesquisas originais para a divulgação dos avanços mais recentes das pesquisas em saúde pública nas Américas" (http://www.scie losp.org/revistas/rpsp/paboutj.htm).

Salud Pública de México: revista bimestral editada pelo Instituto Nacional de Salud Pública, com o objetivo de "disseminar informações sobre saúde pública, compreendida como a aplicação das ciências biológicas, ciências sociais, clínicas e de comportamento para a compreensão dos problemas de saúde da população, assim como para a identificação das necessidades de saúde e para a organização de serviços integrados" (http:/ /www. scielosp.org/revistas/spm/paboutj.htm). 
Nesta análise foram utilizadas as seguintes informações, referentes a 28 de fevereiro de 2003: relação dos periódicos incluídos com as respectivas datas de inclusão, disponíveis no próprio site: número de acessos a home page de cada periódico, número de acessos a sumários e artigos disponíveis no site, referidos ao número de meses de exposição; número mediano e intervalo interquartílico de acessos a fascículos referidos ao número de meses de exposição; média de citações por fascículo e por artigo, disponíveis no site; vida média das citações tomando como ano base o ano de 2002, disponível no site; número e principais periódicos citados nos artigos publicados nos periódicos brasileiros; padrão de citação entre os periódicos da biblioteca eletrônica SciELO Saúde Pública; análise dos resumos dos 50 artigos mais consultados das duas revistas brasileiras avaliando aspectos como língua, tema, método, técnicas de análise e unitermos.

\section{Resultados e discussão}

\section{Acesso livre}

Os acessos mensais, isto é, a média do número de acessos referidos ao número de meses de exposição do periódico variou entre 1.348,77 para Cadernos de Saúde Pública e 231,57 para a Gaceta Sanitaria. A Revista de Saúde Pública recebeu em média 990,83 visitas/mês, ficando na terceira posição. Os acessos aos sumários dos artigos publicados seguiram o mesmo padrão de distribuição. Para Cadernos de Saúde Pública foram registra- dos em média 1.101,52 acessos/mês aos resumos dos artigos publicados, enquanto para a Revista de Saúde Pública foram registrados 916,40 acessos/mês, correspondendo ao segundo e terceiro lugares, ficando atrás somente dos acessos registrados para a Revista Espanhola de Saúde Pública. Entretanto, quando são considerados os acessos aos textos completos dos artigos, as revistas brasileiras passam a ocupar o primeiro e segundo lugares, com 3.743,59 acessos/mês para os artigos publicados nos Cadernos de Saúde Pública e 1.590,97 acessos/mês para os publicados na Revista de Saúde Pública.

Também foi avaliado o número médio de acessos a cada fascículo levando em conta o número de meses de exposição. A Tabela 1 apresenta os fascículos correspondentes ao menor e ao maior número de acessos, valor mediano, primeiro e terceiro quartis, apontando o número médio de consultas/mês de exposição e o conteúdo predominante do fascículo.

Para a Revista de Saúde Pública fica nítido o maior número de consultas/mês de exposição para os fascículos mais recentes, sugerindo que este periódico é consultado principalmente na busca de atualização, pois quanto mais recente a publicação, maior o número de consultas. Para Cadernos de Saúde Pública, embora os fascículos mais consultados também correspondam àqueles mais recentes, a tendência não é tão clara. Os dados parecem indicar que a maior quantidade de acessos é independente da característica temática ou não do fascículo, havendo números temáticos tanto entre os mais consultados como entre os menos consultados, estando a quantidade de consultas, nestes casos, certamente re-

Tabela 1

Número médio de consultas/mês, fascículos correspondentes e conteúdo, por periódico, 1996-2003.

\begin{tabular}{|c|c|c|c|c|c|c|}
\hline \multirow[t]{2}{*}{ Valores } & \multicolumn{3}{|c|}{ Cadernos de Saúde Pública } & \multicolumn{3}{|c|}{ Revista de Saúde Pública } \\
\hline & Consultas/mês & Fascículo & Tema & Consultas/mês & Fascículo & Tema \\
\hline Menor & 35,55 & 15 Suppl 1; 1999 & Bioética & 1,39 & 30(2); 1996 & $\begin{array}{c}\text { Variado } \\
\text { (entomologia) }\end{array}$ \\
\hline Primeiro quartil & 55,81 & 13(2); 1997 & Variado & 37,24 & $32(3) ; 1998$ & Variado \\
\hline Mediana & 156,66 & 15(4); 1999 & Variado & 202,38 & 33(5); 1999 & Variado (idosos) \\
\hline Terceiro quartil & 369,50 & 18 Suppl; 2002 & Eqüidade & 339,58 & 34 Suppl; 2000 & Saúde infantil \\
\hline Maior & $1.617,75$ & 18(5); 2002 & Saúde \& trabalho & $3.450,00$ & $37(1) ; 2003$ & $\begin{array}{c}\text { Variado } \\
\text { (adolescentes) }\end{array}$ \\
\hline
\end{tabular}

Fonte: Centro Latino-Americano e do Caribe de Informação em Ciências da Saúde. 
lacionada ao tamanho da comunidade com interesse nos temas tratados.

O debate, sobre o acesso livre, tem ocupado os editoriais de importantes revistas científicas, dividindo os editores entre os que são favoráveis e aqueles que são contra estas iniciativas 6 . Esses valores dão idéia da possibilidade de disseminação da informação científica através de bibliotecas virtuais que garantam o acesso livre aos textos completos dos artigos, tal como o que ocorre com SciELO Saúde Pública. Em um país de dimensões continentais como o Brasil e, na própria América Latina, onde o financiamento para pesquisa é claramente insuficiente e concentrado em algumas instituições, o acesso livre à informação desempenha papel fundamental no desenvolvimento e consolidação de grupos de pesquisadores 7 .

\section{Índice de citação}

Segundo Forattini (1996, apud Gomes \& Santos 5; p. 91), "para que um periódico seja considerado indispensável à disseminação da informação é necessário que ele, assim como os artigos nele contidos, possuam três atributos: competição (índice de citação); impacto (alcance dos objetivos); internacionalidade (indexação em órgãos especializados)".

Lundberg 8 lembra que o índice de citação é uma medida da extensão pela qual um dado periódico funciona como um elemento de conexão entre pesquisadores em um campo específico do conhecimento. Essa medida foi introduzida há mais de quatro décadas para auxiliar as biblio- tecas a selecionar periódicos científicos a serem adquiridos 9 .

A posição das revistas nacionais no conjunto de periódicos da biblioteca eletrônica pode ser avaliada usando-se dois indicadores: o número médio de citações por fascículo da revista e o número médio de citações por artigo publicado (Tabela 2).

O número de citações é influenciado por uma série de aspectos, dentre os quais se destacam: o campo disciplinar, o tempo de maturação dos artigos, o número médio de autores por artigo, a língua, o período de observação adotado ou “janela”, o número e os tipos de artigos publicados. Para os periódicos considerados, o campo disciplinar é o mesmo, assim como o período de observação adotado. Com relação à língua, quatro periódicos publicam predominantemente em espanhol, um em inglês e outros dois em português. Entretanto, esse fator não parece explicar as diferenças encontradas, pois a média de citações por artigo foi maior para um periódico publicado em português, seguindo-se um periódico predominantemente em inglês e outro em espanhol.

Quanto ao número de artigos publicados, embora exista associação estatisticamente significante entre essas duas variáveis, a média de citações por artigo não parece ser explicada completamente por ela. No período considerado, o maior número de artigos foi publicado pelos Cadernos de Saúde Pública, seguindo-se a Revista Panamericana de Salud Pública e a Revista de Saúde Pública.

Para as revistas brasileiras, as correlações entre o número de fascículos e o número de artigos

Número médio de consultas/mês, fascículos correspondentes e conteúdo, por periódico, 1996-2003.

\begin{tabular}{lcc}
\hline Periódico & Média de citações por fascículo & Média de citações por artigo \\
\hline Bulletin of the World Health Organization & 20,17 & 2,42 \\
Cadernos de Saúde Pública & $38,25\left(2^{\circ}\right)$ & $1,33\left(5^{\circ}\right)$ \\
Gaceta Sanitaria & 15,71 & 1,53 \\
Revista Panamericana de Salud Pública & 7,27 & 0,77 \\
Revista Española de Salud Pública & 9,00 & 1,17 \\
Revista de Saúde Pública & $54,17\left(1{ }^{\circ}\right)$ & $2,83\left(1^{\circ}\right)$ \\
Salud Pública de México & 16,09 & 1,48 \\
\hline
\end{tabular}

Fonte: Centro Latino-Americano e do Caribe de Informação em Ciências da Saúde. 
publicados e o índice de citações são positivas e estatisticamente significantes, com $\mathrm{r}=0,48 \mathrm{e}$ $\mathrm{r}=0,94$, respectivamente.

A série histórica do índice de citações por fascículos e por artigos dos Cadernos de Saúde Pública mostra aumento progressivo entre 1996 e 2005. Para a Revista de Saúde Pública, há praticamente um platô entre 1996 e 2002 e aumento constante entre 2002 e 2005 (Figuras 1 e 2). O índice de citações por fascículos dos Cadernos de Saúde Pública ultrapassa o da Revista de Saúde Pública em 2005, embora por artigo ele permaneça menor. Esse aumento acompanhou o crescimento do número de artigos publicados por ambos os periódicos 10 .

A vida média das citações também é bastante variável e tende a ser cada vez menor na medida da renovação do conhecimento. Um artigo publicado nos Cadernos de Saúde Pública pode esperar ser citado durante em média 3,90 anos, enquanto um artigo publicado na Revista de Saúde Pública pode esperar ser citado em média durante 3,91 anos, ou seja, por praticamente o mesmo tempo. A vida média foi maior para os artigos publicados na Revista Panamericana de Salud Pública $(4,46$ anos), Revista Espanõla de Salud Pública (6,67 anos) e Salud Pública de México (7,21 anos). Tais valores podem estar relacionados com as características dos próprios artigos, esperando-se que pesquisas empíricas tenham menor vida média do que trabalhos de interesse metodológico, conceitual ou teórico.

Os artigos publicados pelos Cadernos de Saúde Pública citaram 742 periódicos diferentes, num total de 2.918 citações. Além de artigos dos próprios Cadernos de Saúde Pública, foram citados artigos da Revista de Saúde Pública, The Lancet, American Journal of Epidemiology, Environmental Health Perspectives e BMJ. Metade das citações concentra-se em 49 periódicos, dos quais 25 são da área de saúde coletiva e 24 de outras áreas das ciências da saúde, sendo 40 periódicos estrangeiros e 9 nacionais. Utilizando-se o critério de classificação dos periódicos adotado pela Coordenação de Aperfeiçoamento de Pessoal de Nível Superior (CAPES), naquele momento, temos 35 periódicos internacionais $\mathrm{A}$, quatro periódicos internacionais $\mathrm{B}$, quatro internacionais

Figura 1

Índice de citações por fascículo publicado. Cadernos de Saúde Pública e Revista de Saúde Pública, 1996-2005.

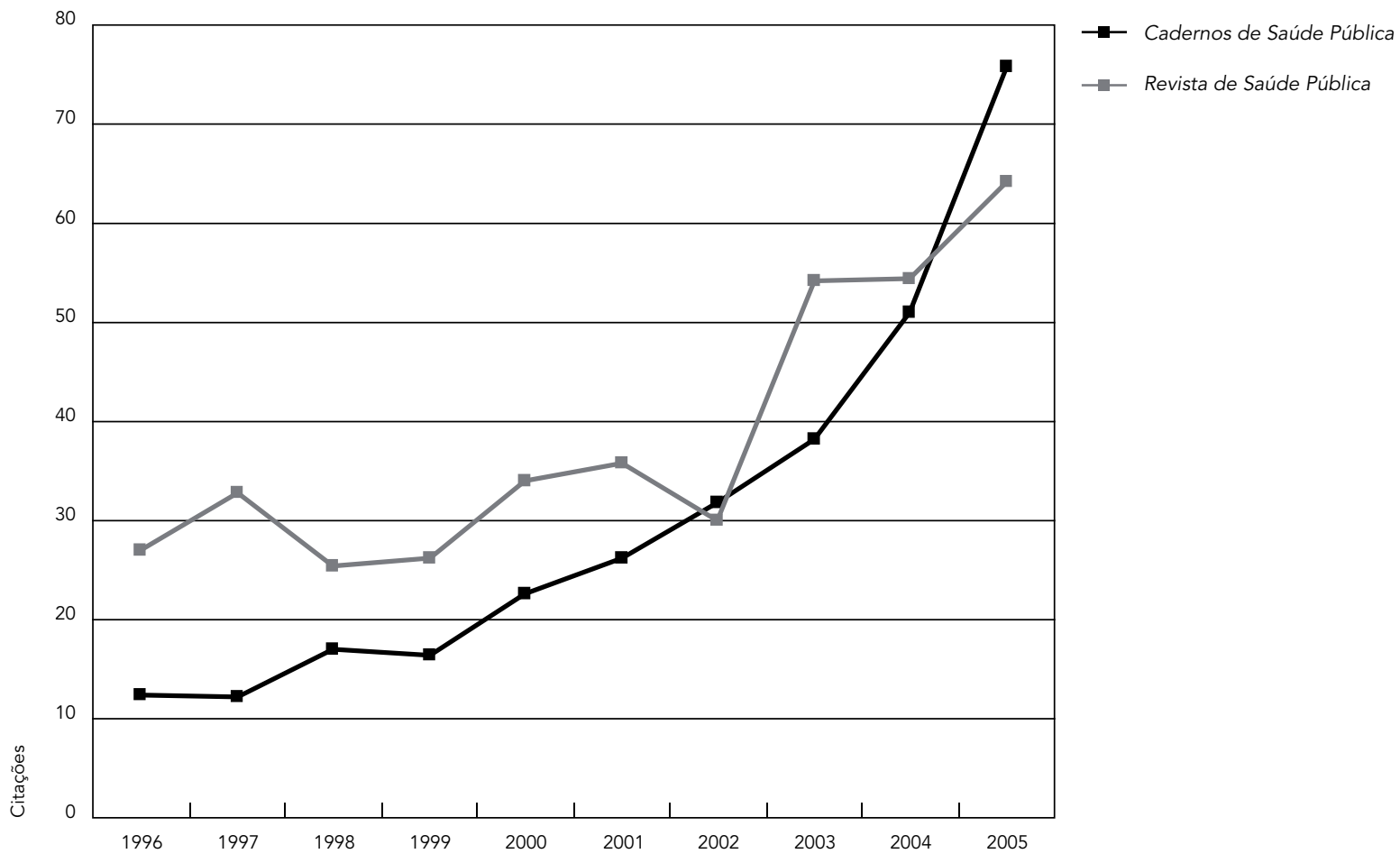




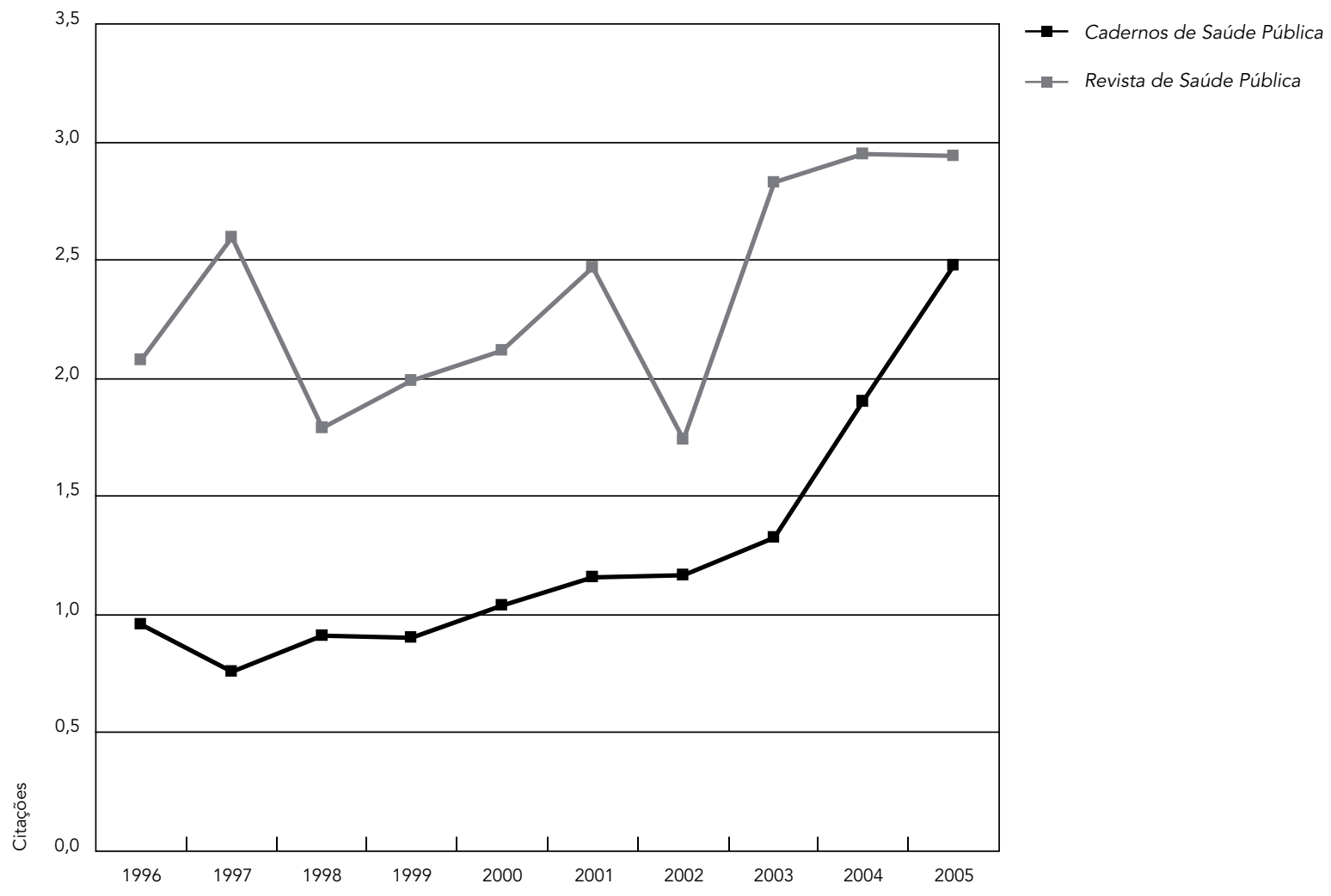

C, três periódicos nacionais A, dois nacionais $\mathrm{B}$ e um periódico nacional C.

Os artigos publicados pela Revista de Saúde Pública citaram 472 periódicos diferentes, 1.390 vezes. Além das citações da própria Revista de Saúde Pública, aparecem por ordem de freqüência das citações: Cadernos de Saúde Pública, The Lancet, JAMA, American Journal of Epidemiology e American Journal of Public Health. Metade das citações concentra-se em 49 periódicos, dos quais 19 são da área de saúde coletiva e 30 de outras áreas das ciências da saúde. Desses 49 periódicos, 42 são estrangeiros e 7 nacionais. Segundo a classificação adotada pela CAPES, 39 são periódicos internacionais $\mathrm{A}$, cinco são internacionais $\mathrm{C}$, um é periódico nacional B e dois são nacionais $\mathrm{C}$.

As citações entre periódicos da biblioteca eletrônica, excluída a autocitação, seguem o seguinte padrão: as revistas brasileiras citamse entre si, ou seja, após a autocitação a revista que mais cita a Revista de Saúde Pública são os
Cadernos de Saúde Pública e vice-versa. O mesmo ocorre entre as revistas espanholas Gaceta Sanitaria e Revista Espanõla de Salud Pública. A Revista Panamericana de Salud Pública e a Salud Pública de México citam mais artigos publicados pelo Bulletin of the World Health Organization e este é mais citado pela Revista de Saúde Pública.

Os "top 50"

Os 50 artigos mais acessados nos Cadernos de Saúde Pública tiveram taxas de acesso por mês de exposição variando entre 6,72 e 52,45. A expressiva maioria desses artigos foi publicada em português (92\%) estando incluídos nessa relação dois artigos publicados em espanhol (4\%) e dois publicados em inglês (4\%). Para a Revista de Saúde Pública, os 50 artigos mais acessados receberam entre 7,33 acessos/mês de exposição e 56,50 acessos/mês. Todos esses artigos foram publicados em português (Figura 3). 


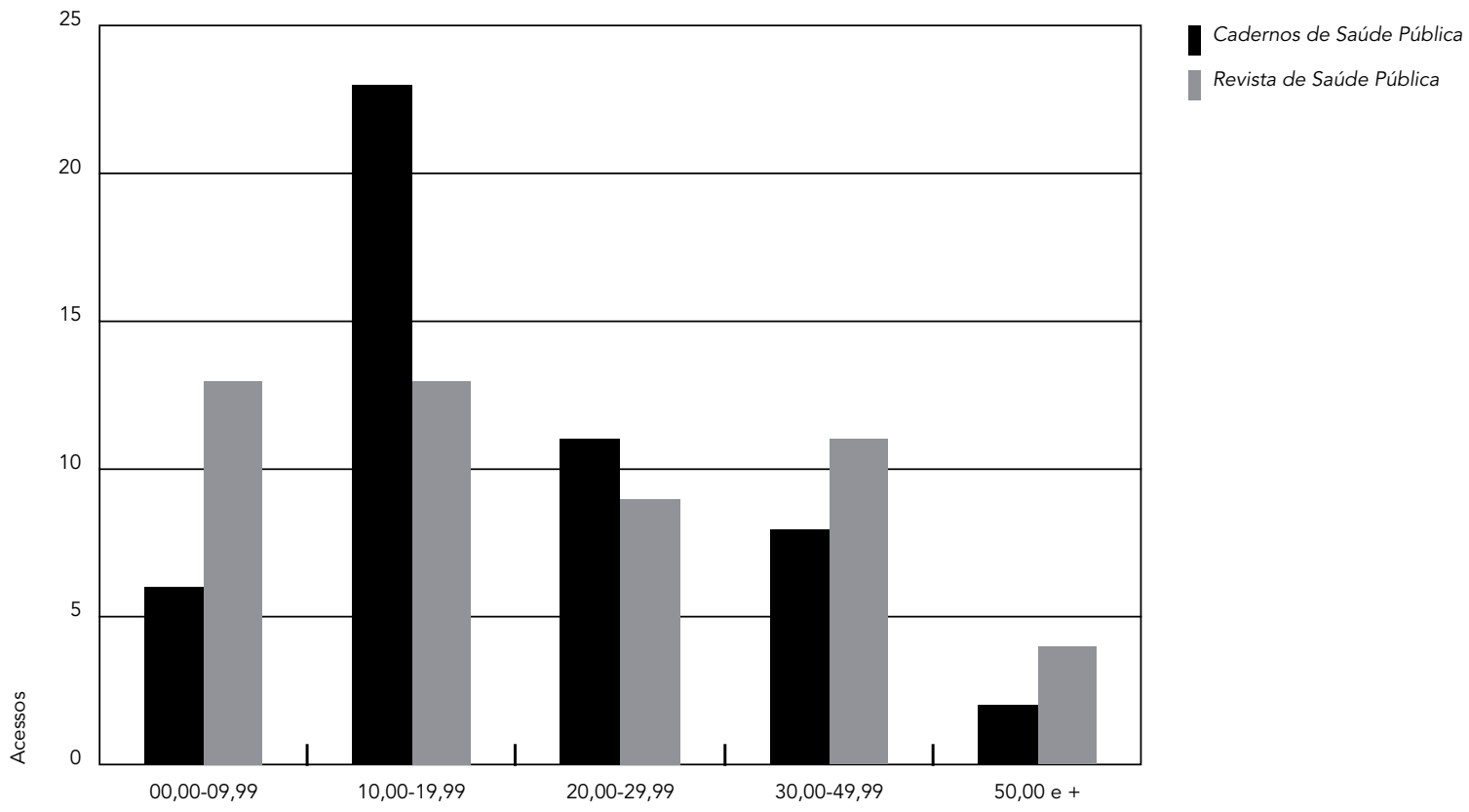

Quanto ao desenho de investigação dentre os 50 artigos mais acessados, $42 \%$ dos publicados nos Cadernos de Saúde Pública eram ensaios teóricos, $20 \%$ eram inquéritos transversais, $16 \%$ eram estudos descritivos e $10 \%$ eram levantamentos em bases de dados nacionais. Os outros seis artigos incluíam estudos caso-controle, ecológico, revisão, intervenção e estudo de casos. Quanto ao desenho utilizado nas investigações que deram origem aos 50 artigos mais acessados na Revista de Saúde Pública, 46\% eram inquéritos transversais, $14 \%$ levantamentos em bases de dados e $12 \%$ revisões bibliográficas. Contrastando com o observado para os Cadernos de Saúde Pública, apenas três artigos eram ensaios teóricos e outros três eram estudos descritivos. Em compensação, apenas um dos artigos dentre os mais acessados nos Cadernos de Saúde Pública era de revisão bibliográfica (Figura 4).

Quanto às técnicas de análise de dados utilizadas nesses artigos, $42 \%$ daqueles publicados nos Cadernos de Saúde Pública eram estudos argumentativos (ensaios teóricos), 34\% quantitativos descritivos, $18 \%$ utilizaram diversas técnicas qualitativas e $6 \%$ a modelagem matemática. Quanto a essa variável, observa-se diferença significante na comparação entre os artigos mais acessados nos dois periódicos, correspondendo aos diferentes desenhos. Assim, os estudos argumentativos (ensaios teóricos) corresponderam a apenas $10 \%$ dos artigos mais acessados na Revista de Saúde Pública, enquanto as técnicas quantitativas descritivas foram utilizadas em $66 \%$ destes artigos. O uso de modelagem matemática foi semelhante ao observado entre os artigos dos Cadernos de Saúde Pública, assim como o uso das técnicas qualitativas (Figura 5).

As áreas disciplinares ou temáticas dos artigos mais acessados foram: saúde e trabalho (50\%), epidemiologia (22\%) e saúde ambiental (8\%), para os artigos publicados nos Cadernos de Saúde Pública. As áreas disciplinares ou temas predominantes nos artigos mais acessados na Revista de Saúde Pública foram: organização de serviços de saúde (22\%), nutrição (22\%), saúde e trabalho (18\%), epidemiologia (12\%) e saúde ambiental (12\%) (Figura 6). Para melhor avaliar até que ponto essas características estão ou não associadas com o maior número de acessos seria preciso analisar uma amostra casual e representativa do universo de artigos disponíveis. A amostra analisada por Carvalho et al. 10 sugere que há acesso proporcional- 
Figura 4

Desenho de estudo dos 50 artigos mais acessados. Revista de Saúde Pública e Cadernos de Saúde Pública, 2003.

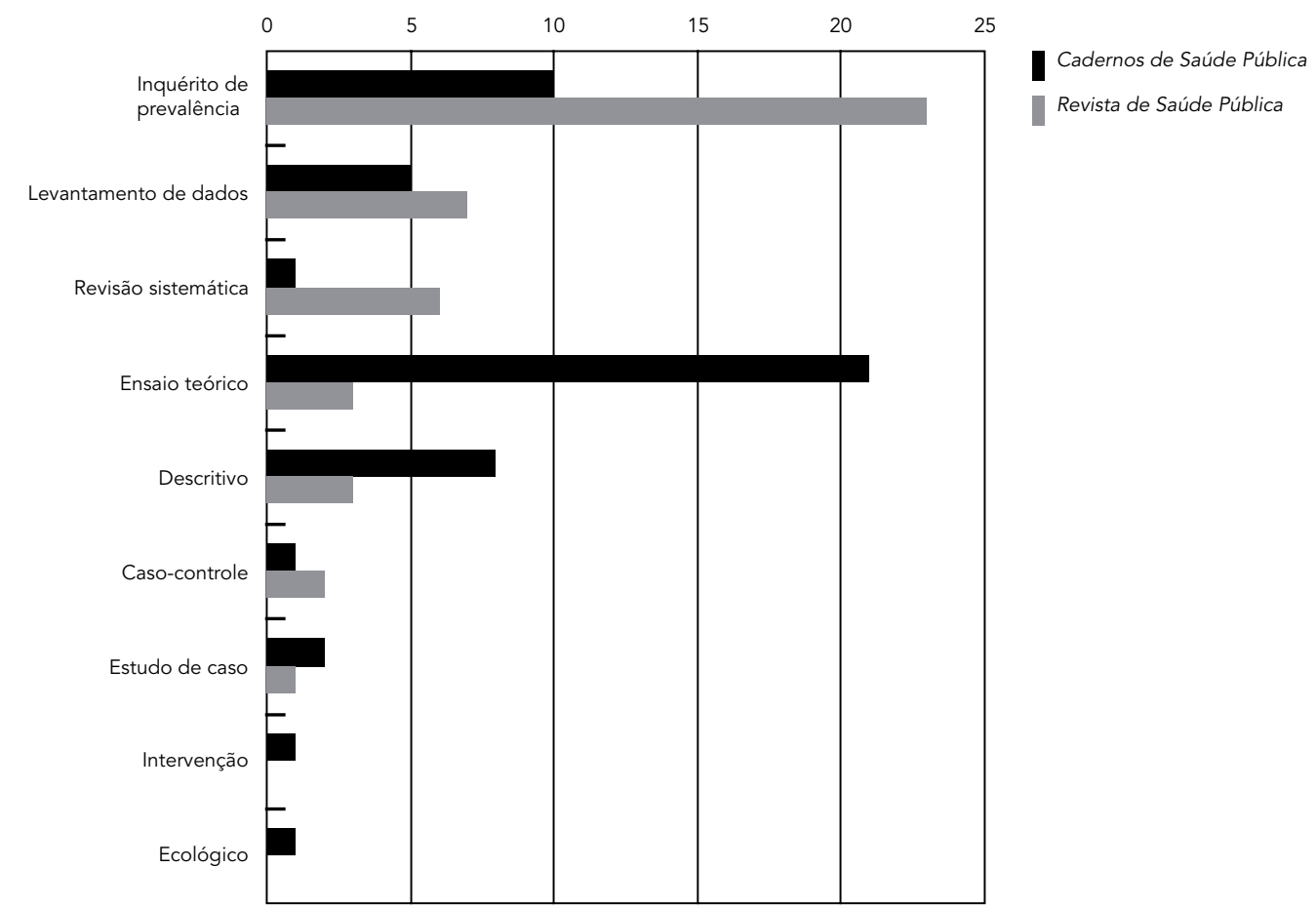

Figura 5

Distribuição das técnicas de análise utilizadas nos artigos mais acessados. Cadernos de Saúde Pública e Revista de Saúde Pública, 2003.

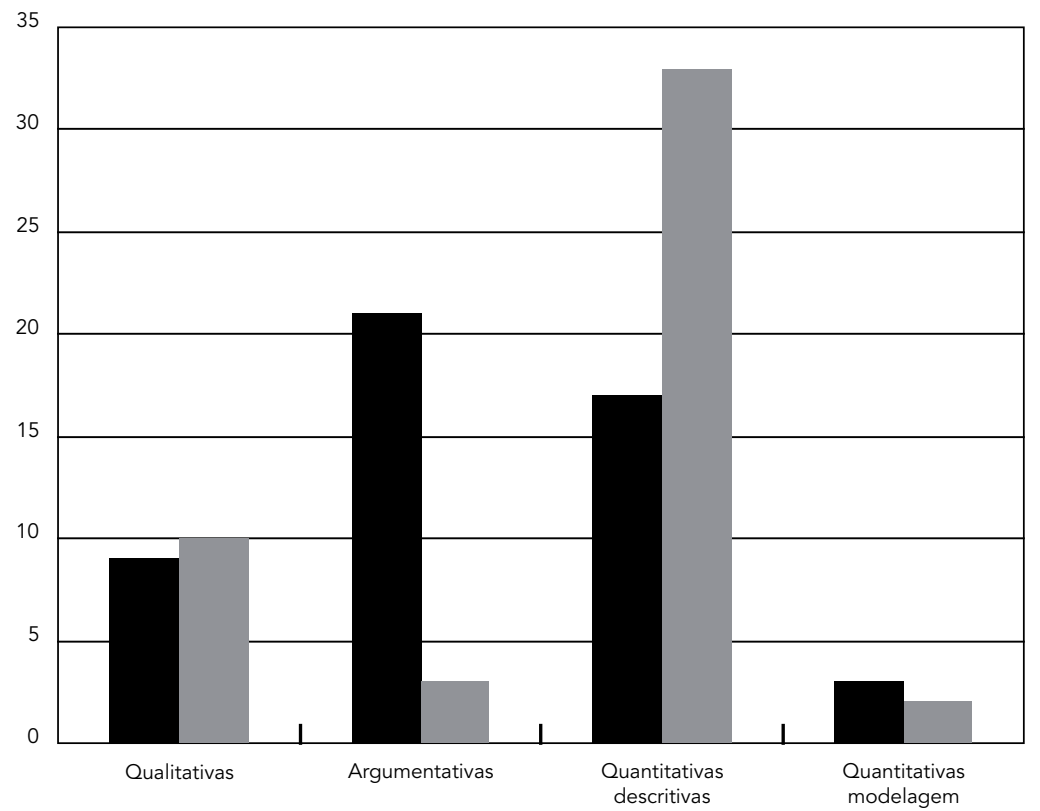

Cadernos de Saúde Pública

Revista de Saúde Pública 


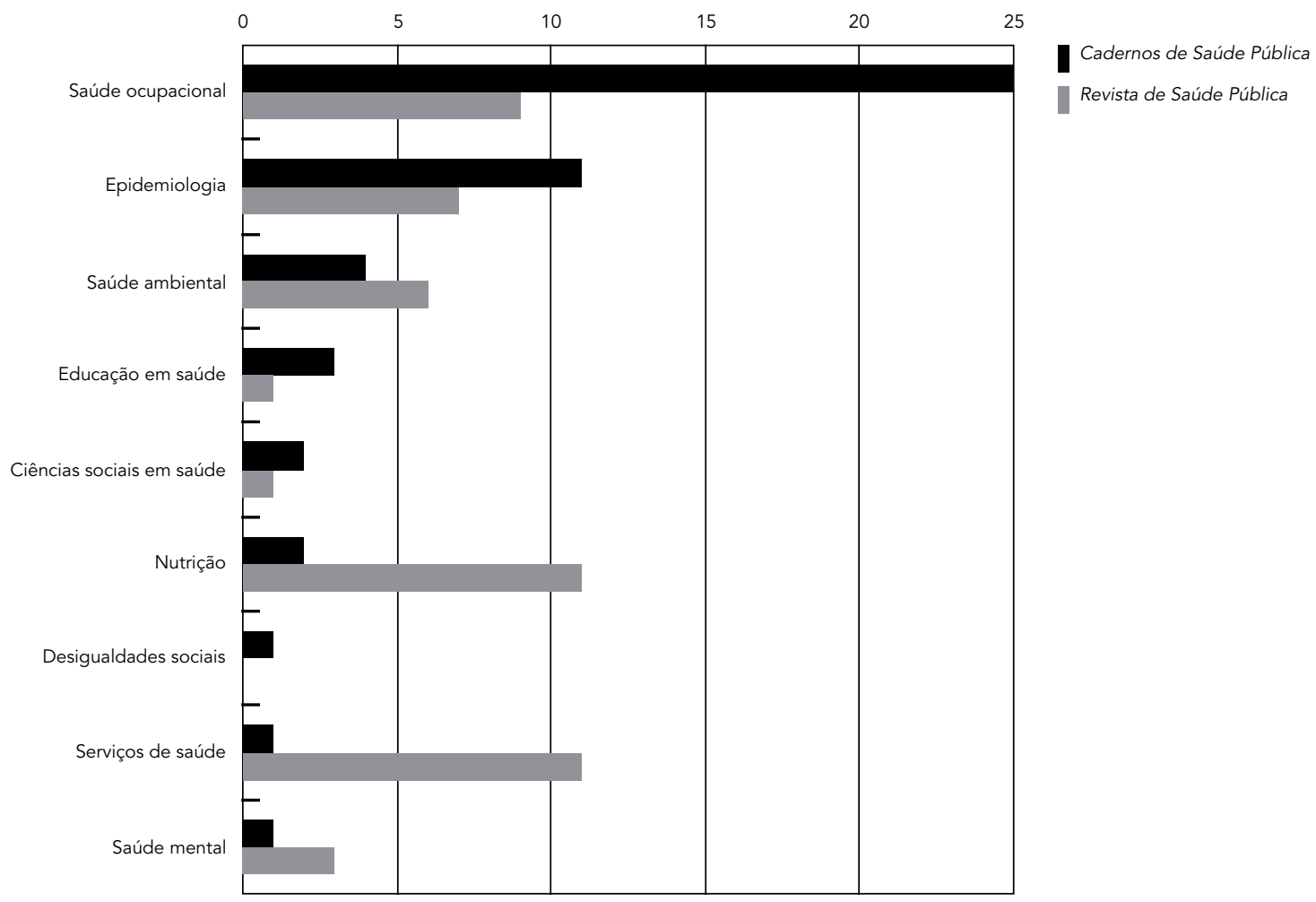

mente maior a artigos referentes a serviços de saúde e menor para artigos de epidemiologia.

As maiores diferenças na distribuição das temáticas foram observadas para três áreas: saúde ocupacional, responsável pela metade dos artigos mais acessados nos Cadernos de Saúde Pública e, 20\% na Revista de Saúde Pública; nutrição correspondendo a $22 \%$ dos artigos mais acessados na Revista de Saúde Pública e apenas 4\% nos Cadernos de Saúde Pública; planejamento e avaliação de programas e ações de saúde, incluindo questões relativas à prática profissional em saúde referente a $22 \%$ dos artigos mais acessados na Revista de Saúde Pública e apenas 2\% nos Cadernos de Saúde Pública.

\section{Conclusões}

Os dados apresentados ressaltam a importância da SciELO Saúde Pública para a disseminação da informação científica na América Latina. Os periódicos brasileiros nela incluídos vêm apresentando desempenho satisfatório tanto no sentido do número de acessos que recebem quanto nos indicadores cientométricos.

Essas informações podem ser úteis aos editores e revisores de ambos os periódicos, fornecendo o perfil dos artigos mais acessados e orientando a política editorial.

Da mesma maneira podem auxiliar os autores na identificação do perfil da publicação para a qual encaminhar o resultado de sua produção, bem como os leitores, na busca de informações científicas de interesse.

Finalmente, os indicadores cientométricos podem e devem ser utilizados para a qualificação da produção nacional, com maior propriedade do que indicadores produzidos por outras bases de indexação, na medida em que podem refletir a disseminação da informação na América Latina e no Brasil, sem as limitações impostas pela língua e para um campo específico do conhecimento. Os indicadores produzidos por outras bases estrangeiras poderão, evidentemente, continuar a ser usados como indicativos da difusão científica em países europeus e norteamericanos. 


\section{Resumo}

O objetivo deste artigo é analisar dois periódicos brasileiros incluídos na biblioteca SciELO Saúde Pública, por meio de um conjunto de indicadores e análise dos artigos mais acessados. Cadernos de Saúde Pública: 3.743,59 acessos/mês, média de 30,31 citações por artigo. Os 50 mais acessados (6,72 a 524,50 acessos) foram publicados em português (92\%), $42 \%$ eram ensaios teóricos, $20 \%$ inquéritos transversais, $16 \%$ estudos descritivos. $42 \%$ usaram técnicas argumentativas, 34\% quantitativas, $18 \%$ técnicas qualitativas e $6 \%$ modelagem matemática. As áreas temáticas mais contempladas foram: saúde e trabalho (50\%), epidemiologia (22\%) e saúde ambiental (8\%). Revista de Saúde Pública: 1.590,97 acessos/mês, com média de 26,27 citações por artigo. Os 50 mais acessados (7,33 e 56,50 acessos) foram todos publicados em português, $46 \%$ eram inquéritos transversais, $14 \%$ levantamentos em bases de dados e $12 \%$ revisões bibliográficas. As técnicas quantitativas foram utilizadas em $66 \%$ desses artigos, modelagem matemática foi semelhante ao observado entre os artigos dos Cadernos de Saúde Pública, assim como as técnicas qualitativas. Os temas predominantes foram: organização de serviços de saúde (22\%), nutrição (22\%), saúde e trabalho (18\%), epidemiologia (12\%) e saúde ambiental (12\%).

Acesso Aberto; Publicações Periódicas; Bibliotecas Virtuais; Índices

\section{Referências}

1. Rasslan S, Barata RB, Rodrigues JJG. Pós-graduação, produção intelectual e veículo de publicação. Rev Col Bras Cir 2003; 30:1-3.

2. Greenhalg T. Como ler artigos científicos. Porto Alegre: Editora Artmed; 2005.

3. Malforms B, Gamsworthy P, Grossman M. Writing and presenting scientific papers. Nottingham: Nottingham University Press; 2000.

4. Volpato GL, Freitas EG. Desafios na publicação científica. Pesqui Odontol Bras 2003; 17 Suppl 1:49-56.

5. Gomes SP, Santos MALC. Avaliação de um periódico na área de medicina tropical. Ciênc Inf 2001; 30:91-100.

6. Delamothe T, Godlee F, Smith R. Scientific literature's open sesame? BMJ 2003; 326:945-6.

7. Meis L, Velloso A, Lannes D, Carmo MS, Meis C. The growing competition in Brazilian science: rites of passage, stress and burnout. Braz J Med Biol Res 2003; 36:1135-41.

8. Lundberg GD. The "omnipotent" science citation index impact factor. Med J Aust 2003; 178:253-4.

9. Walter G, Bloch S, Hunt G, Fisher K. Counting on citations: a flawed way to measure quality. Med J Aust 2003; 178:280-1.

10. Carvalho L, Coimbra Jr. CEA, Souza-Santos R, Santos RV. Produção e citação em Saúde Coletiva: um olhar a partir dos periódicos Cadernos de Saúde Pública e Revista de Saúde Pública. Cad Saúde Pública 2007; 23:3023-30.

Recebido em 28/Jun/2006

Aprovado em 01/Jun/2007 